\title{
AN ALGORITHM FOR MADM BASED ON SUBJECTIVE PREFERENCE
}

Feng Kong, Hongyan Liu

Department of Economics and Management, North China Electric Power University, Baoding, P.R.China, 071003

Abstract: In multi-attribute decision making (MADM) problems, as human judgments are often vague and complex, it is usually difficult for decision makers to estimate an exact or crisp value for his preferences. Further, the decision maker's subjective preferences affect the decision result directly. However, there are only a few researches on fuzzy MADM which took into account the decision maker's preferences. This paper put forward a new algorithm for fuzzy MADM, which takes into account the decision maker's preferences. This method makes our new algorithm more reasonable and the results in more agreement with the decision maker's intentions.

Key words: Fuzzy MADM; subjective preference; fuzzy weights; ideal solution

\section{INTRODUCTION}

In Multi-attribute Decision Making (MADM) problems, the decision maker is often faced with the problem of selecting among alternatives that have conflicting attributes. For example, in site selection problems, attributes such as prices, transportation, costs of operations, and the prospects of products are often considered in the selection of a satisfactory place. For some of these attributes, we can give exact assessments; while for others, we cannot. Since human judgments and preferences are often vague and complex, and decision makers cannot estimate their preferences with an exact scale, we can only give linguistic assessments instead of exact assessments. So fuzzy set theory is introduced into MADM, Fuzzy MADM is put forward to solve such uncertainty problems. 
No matter what method is used to solve Fuzzy MADM problems, weights of attributes reflecting the relative importance of the attributes must be determined. There are many approaches to determine the weights of attributes. We can divide those approaches into subjective approaches and objective ones. The former selects weights according to the preference information of decision makers, such as the eigenvector method, weighted least square method, Delphi method, and mathematical programming models etc. The latter determines weights by solving mathematical models automatically without any consideration of the decision maker's preferences, for example, the entropy method, multiple objective programming, etc. Weights determined by subjective approaches can reflect the subjective judgments of decision makers, thus makes the rankings of alternatives in Fuzzy MADM problem have more arbitrary factors. The objective approaches select weights through mathematical calculation, which neglects subjective judgment information of decision makers. Since either subjective approaches or objective approaches have their advantages and disadvantages, an integrated or combined method seems more desirable in the determination of attribute weights. Thus, this paper tries to give a new weight determination approach to retain the merits of both subjective and objective approaches: to determine weights by solving mathematical models automatically and at the same time take into consideration the decision maker's preferences.

In this paper, the weights of attributes and the assessments of alternatives are described as fuzzy numbers. We put forward a new approach, which integrated the subjective and objective information, to determine the weights of attributes. In Section 2, we will give some introductions to fuzzy numbers. In Section 3, a combined approach to determine attributes weights is put forward. An algorithm for fuzzy MADM based on decision maker's subjective ideal solution is explored in detail and numerical example is illustrated in Section 4, with the conclusion in Section 5.

\section{FUZZY NUMBERS}

The fuzzy sets theory, introduced by Zadeh (1965) to deal with vague, imprecise and uncertain problems, has been used as a modeling tool for complex systems that can be controlled by humans but are hard to define precisely.

In this paper, we use triangular fuzzy numbers to illustrate our approach, though our approach fit with all types fuzzy numbers. Triangular fuzzy number is a special kind of fuzzy sets. A triangular fuzzy number can be 
denoted as: $N=(a, b, c)$. The membership function of triangular fuzzy numbers is:

$$
\mu(x)= \begin{cases}\frac{x-a}{b-a}, & a \leq x \leq b ; \\ \frac{c-x}{c-b}, & b \leq x \leq c ; \\ 0, & \text { else }\end{cases}
$$

Particularly, when $a=b=c$, trapezoidal fuzzy numbers become crisp numbers. That is, crisp numbers can be considered as a special case of fuzzy numbers.

The left and right fuzzy sets of a fuzzy number, $N$, can be denoted respectively as $N_{L}$ and $N_{R}$, and their membership functions are respectively defined as:

$$
\begin{aligned}
& \mu_{N_{l}}(x)=\sup _{x=y+z, z \leq 0} \mu_{N}(y) \\
& \mu_{N_{R}}(x)=\sup _{x=y+z, z \geq 0} \mu_{N}(y)
\end{aligned}
$$

Given any two fuzzy numbers $\tilde{N}=\left(a_{1}, b_{1}, c_{1}\right), \tilde{M}=\left(a_{2}, b_{2}, c_{2}\right)$ and a real number $\lambda$, some main operations of fuzzy numbers can be expressed as follows:

$$
\begin{aligned}
& \tilde{N}+\tilde{M}=\left(a_{1}+a_{2}, b_{1}+b_{2}, c_{1}+c_{2}\right) \\
& \tilde{N} \times \tilde{M}=\left(a_{1} \times a_{2}, b_{1} \times b_{2}, c_{1} \times c_{2}\right) \\
& \tilde{N}^{\lambda}=\left(a_{1}^{\lambda}, b_{1}^{\lambda}, c_{1}^{\lambda}\right)
\end{aligned}
$$

\section{A COMBINED APPROACH TO DETERMINE ATTRIBUTES WEIGHTS}

Classical Fuzzy MADM problems usually assume that there are $m$ feasible alternatives, $A_{1}, A_{2}, A_{3}, \ldots$, and $A_{m}$, with each of the alternatives having $n$ attributes, $f_{1}, f_{2}, f_{3}, \ldots$, and $f_{n}$, and the weight of each attribute being $w_{1}, w_{2}, w_{3}, \ldots$, and $w_{n}$. The goal of decision-making is to find the most satisfactory one among the $\mathrm{m}$ alternatives. The decision problem can be expressed as the following: 


$$
\begin{aligned}
D & =\left[\begin{array}{cccc}
y_{11} & y_{12} & \ldots & y_{1 n} \\
y_{21} & y_{22} & \ldots & y_{2 n} \\
\ldots & \ldots & \ldots & \ldots \\
y_{m 1} & y_{m 2} & \ldots & y_{m n}
\end{array}\right] \\
W & =\left(w_{1}, w_{2}, \ldots, w_{n}\right)
\end{aligned}
$$

where $y_{i j}$ represents the uncertain assessments of the $j$-th attribute of the $i$-th alternative. They can be either fuzzy numbers or linguistic words.

Assume that all values for each attribute are scaled between 0 and 1 to have the same range of measurement. This is achieved by normalizing elements in the decision matrix using the following formulas:

For benefit criteria, $y_{j}^{+} \geq \sup \left(\widetilde{y}_{1 j}, \tilde{y}_{2 j}, \ldots, \tilde{y}_{m j}\right) ; y_{j-}^{-} \leq \inf \left(\widetilde{y}_{1 j}, \tilde{y}_{3 j}, \ldots, \widetilde{y}_{m j}\right)$.

$$
\widetilde{x}_{i j}=\left(\frac{a_{i j}-y_{j}^{-}}{y_{j}^{+}-y_{j}^{-}}, \frac{b_{i j}-y_{j}^{-}}{y_{j}^{+}-y_{j}^{-}}, \frac{c_{i j}-y_{j}^{-}}{y_{j}^{+}-y_{j}^{-}}\right), i=1,2, \ldots, m
$$

For cost type criteria, $y_{j}^{+} \leq \inf \left(\widetilde{y}_{1 j}, \tilde{y}_{3 j}, \ldots, \tilde{y}_{m j}\right) ; y_{j-}^{-} \geq \sup \left(\tilde{y}_{1 j}, \tilde{y}_{3 j}, \ldots, \tilde{y}_{m j}\right)$.

$$
\widetilde{x}_{i j}=\left(\frac{y_{j}^{+}-c_{i j}}{y_{j}^{-}-y_{j}^{+}}, \frac{y_{j}^{+}-b_{i j}}{y_{j}^{-}-y_{j}^{+}}, \frac{y_{j}^{+}-a_{i j}}{y_{j}^{-}-y_{j}^{+}}\right), i=1,2, \ldots, m
$$

where, $\widetilde{x}_{i j}$ represents the scale in the decision matrix after normalization, which belongs to $[0,1]$. Therefore, after normalization, the values of each criterion of both the ideal solution and negative ideal solution are respectively: $x_{j}^{+}=(1,1,1)=1$, and $\overline{x_{j}^{-}}=(0,0,0)=0$.

The linguistic terms can be expressed in corresponding triangular fuzzy numbers as Table 1 show ${ }^{[3][4][5][6]}$.

Table 1. Linguistic terms and triangular fuzzy numbers

\begin{tabular}{ll}
\hline Linguistic terms & Corresponding Fuzzy numbers \\
\hline Very bad (VB) & $(0,0,0.1)$ \\
Bad (B) & $(0,0.1,0.3)$ \\
Medium bad (MB) & $(0.1,0.3,0.5)$ \\
Medium (M) & $(0.3,0.5,0.7)$ \\
Medium good (MG) & $(0.5,0.7,0.9)$ \\
Good (G) & $(0.7,0.9,1.0)$ \\
Very good (VG) & $(0.9,1.0,1.0)$ \\
\hline
\end{tabular}




\subsection{Determination of Subjective Weights}

Suppose the decision maker give his pairwise comparison vector, $V$, on the attribute set ${ }^{[1][10][11][12][13][14]}$.

$$
V=\left(v_{11}, v_{12}, \ldots, v_{1 n}\right)
$$

where, $v_{1 j}$ is a fuzzy number comparison scale, $v_{1 j}=\left(v_{1 j}^{l}, v_{1 j}^{m}, v_{1 j}^{r}\right) \in[0,1]$, which represent the relative importance of the first attribute with respect to the $j$-th attribute. The fuzzy comparison scales can be obtained from the decision maker.

There is $v_{11}=(0.5,0.5,0.5)$. The first attribute is considered to be of equal importance with the $j$-th attribute, if $v_{1 j}=0.5$; the first attribute is more important than the $j$-th attribute, if $v_{1 j}>0.5$; and the first attribute is not so important as the $j$-th attribute, if $v_{1 j}<0.5$.

Using the fuzzy numbers normalization method of Dubois and Prade (1982) ${ }^{[9]}$, calculate the subjective weights of the attributes:

$$
\begin{aligned}
& W_{j}^{s}=\left(W_{j}^{s l}, W_{j}^{s m}, W_{j}^{s r}\right), j=1,2, \ldots, n \\
& W_{j}^{s m}=\frac{\frac{1}{v_{1 j}^{m}}-1}{\sum_{k=1}^{n}\left(\frac{1}{v_{1 k}^{m}}-1\right)}, k=1,2, \ldots, n \\
& W_{j}^{s l}=\frac{\frac{1}{v_{1 j}^{l}}-1}{\frac{1}{v_{1 j}^{l}}-1+\sum_{k \neq j}\left(\frac{1}{v_{1 k}^{r}}-1\right)}, \\
& W_{j}^{s r}=\frac{\frac{1}{v_{1 j}^{r}}-1}{\frac{1}{v_{1 j}^{r}}-1+\sum_{k \neq j}\left(\frac{1}{v_{1 k}^{l}}-1\right)} .
\end{aligned}
$$

\subsection{Determination of Objective Weights}

As we know, entropy theory is another important theory to study the problem of uncertainty. Entropy weight is a parameter that describes how 
much different alternatives approach one another in respect to a certain attribute. The greater the value of the entropy, the smaller the entropy weight, then the smaller the differences of different alternatives in this specific attribute, and the less information the specific attribute provides, and the less important this attribute becomes in the decision making process. In this paper we give a Fuzzy Entropy Weight.

For crisp numbers, the calculation of the entropies is very straightforward. Usually we will use the following formula :

$$
E(i)=-K \sum_{i=1}^{m} x_{i j} \ln x_{i j}
$$

where $K$ is a constant.

While for fuzzy numbers, we could not use the above formula to calculate the entropies of fuzzy numbers directly. Generally, we would first transform the fuzzy numbers into crisp numbers, and then calculate their respective entropies. Although there are many methods to transform fuzzy numbers, most of these methods did not take into account the decisionmaker's preferences for the degree of uncertainties. Kong $(2004)^{[20]}$ gave a formula that took into account these factors:

$$
\begin{gathered}
F\left(x_{i j}\right)=m\left(x_{i j}\right)-\beta \sigma\left(x_{i j}\right) \\
m\left(x_{i j}\right)=\frac{\int x \mu\left(x_{i j}\right) d x}{\int \mu\left(x_{i j}\right) d x}, \sigma\left(x_{i j}\right)=\left[\frac{\int x^{2} \mu\left(x_{i j}\right) d x}{\int \mu\left(x_{i j}\right) d x}-m^{2}\left(x_{i j}\right)\right]^{1 / 2} ;
\end{gathered}
$$

where $F\left(x_{i j}\right)$ represents the ranking index of the $j$-th attribute of the $i$-th alternative, $\beta$ represents the decision-maker's uncertainty-aversion coefficient, when $\beta>0$, the decision maker is uncertainty-averse, when $\beta<0$, the decision maker is uncertainty-loving, and when $\beta=0$, the decision maker is uncertainty-neutral.

Next, normalize $F\left(x_{i j}\right)$ according to the following equation:

$$
f_{i j}=\frac{F\left(x_{i j}\right)}{\sum_{i=1}^{m} F\left(x_{i j}\right)}
$$

Then, the fuzzy entropies of the attributes can be calculated with the following: 


$$
\widetilde{E}_{j}=-K \sum_{i=1}^{m} f_{i j} \ln f_{i j}=-\frac{1}{\ln m} \sum_{i=1}^{m} f_{i j} \ln f_{i j} .
$$

Now, calculate the fuzzy entropy weight with the following equation:

$$
W_{j}^{o}=\frac{\left(1-\widetilde{E}_{j}\right)}{\sum_{j=1}^{n}\left(1-\widetilde{E}_{j}\right)}
$$

\subsection{Calculation of the Combined Fuzzy Weights}

Derive the combined fuzzy weight according to:

$$
W_{j}=\left(W_{j}^{s}\right)^{\alpha} \cdot\left(W_{j}^{o}\right)^{\gamma}
$$

where $\alpha$, and $\gamma$ represents the relative importance of the subjective weights and the objective weights to decision makers respectively, $\alpha+\gamma=1$.

Combined fuzzy weight tells us that, if the values of an attribute in different alternatives do not differ much from one another, then the attribute will not be so important in the decision making process, though this attribute may seem very important to the decision-maker. Only those attributes which are both important to the decision-maker and whose values in different alternatives differ significantly can play important roles in decision-making processes. Combined fuzzy weight is such an indicator that not only shows how much important an attribute is to the decision-maker, but also shows how much different the values of the attribute in different alternatives are.

\section{THE NEW ALGORITHM FOR FUZZY MADM BASED ON SUBJECTIVE PREFERENCES}

\subsection{Algorithm for MADM}

Considering the different importance of each attribute, we should assign corresponding weights to the normalized fuzzy decision matrix to make it become: $\widetilde{R}=\left[\widetilde{r}_{i j}\right]_{m \times n}$, where

$$
\widetilde{r}_{i j}=\widetilde{x}_{i j} \times \widetilde{w}_{j}
$$


and the weighted values of each attribute of both the ideal solution and negative ideal solution respectively is:

$$
\widetilde{r}_{j}^{+}=x_{j}^{+} \times \widetilde{w}_{j}=\widetilde{w}_{j}, \widetilde{r}_{j}^{-}=x_{j}^{-} \times \widetilde{w}_{j}=0 .
$$

It is obvious that, the more the value of each attribute approaches the value of the ideal solution, and at the same time, the more faraway they are from the negative ideal solution, the better the alternative is. In fuzzy MADM, this approach can be represented by the distance between the crisp numbers (the values of ideal solution and negative ideal solution) and fuzzy numbers (the values of criteria of alternative), and it is affected by the decision maker's subjective preference.

The distance between each criterion of an alternative and the corresponding one of the ideal solution, can be derived according to the weighted Hamming fuzzy distance:

$$
d_{i j}^{+}=\rho d_{H}\left(r_{j I}^{+}, r_{i j L}\right)+(1-\rho) d_{H}\left(r_{j R}^{+}, r_{i j R}\right)
$$

where $d_{H}$ represents the relative Hamming distance of the fuzzy set, $\rho$ represents the coefficient of the decision maker's uncertainty preference, $0 \leq \rho \leq 1$. When $\rho>0.5$, the decision maker is uncertainty-averse, when $\rho<0.5$, the decision maker is uncertainty-loving, and when $\rho=0.5$, the decision maker is uncertainty-neutral.

The same, we can derive the distance between the same criteria of the negative ideal solution and each alternative:

$$
d_{i j}^{-}=\rho d_{H}\left(r_{j L}^{-}, r_{i j L}\right)+(1-\rho) d_{H}\left(r_{j L}^{-}, r_{i j R}\right)
$$

The distance between each alternative and the ideal solution is given by:

$$
D_{i}^{+}=\left\|\left(d_{i 1}^{+}, d_{i 2}^{+}, \ldots, d_{i n}^{+}\right)\right\|=\sqrt{\sum_{j=1}^{n}\left(d_{i j}^{+}\right)^{2}}
$$

and the distance between each alternative and the negative ideal solution is given by:

$$
D_{i}^{-}=\left\|\left(d_{i 1}^{-}, d_{i 2}^{-}, \ldots, d_{i n}^{-}\right)\right\|=\sqrt{\sum_{j=1}^{n}\left(d_{i j}^{-}\right)^{2}}
$$


A closeness coefficient is defined to determine the ranking order of all alternatives. The closeness coefficient of each alternative is calculated by:

$$
C_{i}=\frac{D_{i}^{+}}{D_{i}^{+}+D_{i}^{-}}
$$

The bigger its value, the better the alternative.

\subsection{A numerical illustration}

Now a firm has four feasible alternative plans, $A_{1}, A_{2}, A_{3}$ and $A_{4}$, to develop a new product. The firm evaluates each of the four alternatives in four aspects, risk, profit, prospect, and development. See the following Table 2 for the details of the evaluations.

Table. 2 Evaluation of the four alternatives

\begin{tabular}{lllll}
\hline & Risk & $\begin{array}{l}\text { Profit } \\
(10,000 \$)\end{array}$ & Prospect & Development \\
\hline$A_{1}$ & High & $(80,90,100)$ & Medium & Very difficult \\
$A_{2}$ & Very low & $(20,30,40)$ & Medium & Very easy \\
$A_{3}$ & Medium & $(60,75,80)$ & Good & Difficult \\
$A_{4}$ & Low & $(80,85,95)$ & Very Good & Easy \\
\hline
\end{tabular}

Now we show the steps of the decision-making.

Step 1: The decision maker gives his pairwise comparison vector, $\mathrm{V}$, on the attributes,

$$
\begin{aligned}
& V=\left(v_{11}, v_{12}, v_{13}, v_{14}\right) \\
& v_{11}=(0.5,0.5,0.5) ; v_{12}=(0.4,0.45,0.5) ; \\
& v_{13}=(0.7,0.75,0.8) ; v_{14}=(0.5,0.55,0.6)
\end{aligned}
$$

Then calculate the subjective fuzzy weights of attributes according to equations (5) (7),

$$
\begin{aligned}
& W_{1}^{s}=(0.252,0.271,0.289) ; W_{2}^{s}=(0.269,0.301,0.337) ; \\
& W_{3}^{s}=(0.161,0.181,0.201) ; W_{4}^{s}=(0.219,0.247,0.276) .
\end{aligned}
$$

Step 2: Calculate the objective weights.

First, transform the fuzzy linguistic terms into triangular fuzzy numbers according to Table 1 . Then derive the normalized decision matrix as in Table 3. Suppose, $y_{2}^{+}=1000000$ and $y_{2}^{-}=0$. 
Table 3 The normalized fuzzy decision matrix

\begin{tabular}{lllll}
\hline & Risk & Profit & Prospect & Development \\
\hline & $(0.9,1,1)$ & $0.8,0.9,1)$ & $(0.3,0.5,0.7)$ & $(0,0.1,0.1)$ \\
$A_{2}$ & $(0,0.1,0.1)$ & $(0.2,0.3,0.4)$ & $(0.3,0.5,0.7)$ & $(0.9,1,1)$ \\
$A_{3}$ & $(0.3,0.5,0.7)$ & $(0.6,0.75,0.8)$ & $(0.7,0.9,1)$ & $(0,0.1,0.3)$ \\
$A_{4}$ & $(0,0.1,0.3)$ & $(0.8,0.85,0.95)$ & $(0.9,1,1)$ & $(0.7,0.9,1)$ \\
\hline
\end{tabular}

Then calculate the objective weights according to equations (9) (12), let $\beta=2$.

$$
W_{1}^{o}=0.465, W_{2}^{o}=0.061, W_{3}^{o}=0.064, W_{4}^{o}=0.409 \text {. }
$$

Step 3: Calculate the fuzzy combined weights by equation (13), we get:

$$
\begin{aligned}
& W_{1}=(0.342,0.355,0.367) ; W_{2}=(0.128,0.136,0.144) \\
& W_{3}=(0.102,0.105,0.114) ; W_{4}=(0.299,0.318,0.336) .
\end{aligned}
$$

Step 4: Calculate closeness of the alternatives according to equation (19) (24), $\rho=0.7$ :

$$
C_{1}=0.584 ; C_{2}=0.279 ; C_{3}=0.473 ; C_{4}=0.437 \text {. }
$$

Thus the order of the alternatives is: $A_{1} \succ A_{3} \succ A_{4} \succ A_{2}$. That is, alternative $A_{1}$ is the optimal one.

\section{CONCLUSIONS}

Fuzzy sets theory can be applied to deal with multi-attribute decision making problems under uncertainty and imprecise environment. In this paper, we made some improvement on the fuzzy MADM method by taking into account the decision maker's subjective preferences. This method makes our new algorithm more reasonable and the results in more agreement with the decision maker's intentions.

\section{ACKNOWLEDGEMENTS}

This paper is supported by Doctorial Foundation of North China Electric Power University. 


\section{REFERENCES}

[1] C.L. Hwang, K. Yoon. Multiple criteria decision making method and applications. Springer, Berlin Heidelberg, 1981.

[2] M. Delgado, F. Herrera, et al. Combining numerical and linguistic information in group decision making. Journal of information sciences, 1998, 107: 177 194.

[3] Ching-Hsue Cheng, Yin Lin. Evaluating the best main battle tank using fuzzy decision theory with linguistic criteria evaluation. European Journal of Operational Research, 2002, 142: $174 \sim 186$.

[4] F. Herrera, E. Herrera-Viedma, J.L. Verdegay. Linguistic Measures Based on Fuzzy Coincidence for Reaching Consensus in Group Decision Making. International Journal of Approximate Reasoning, 1997, 16: 309 334.

[5] Chen-Tung Chen. Extension of the TOPSIS for group decision making under fuzzy environment". Fuzzy Sets and Systems, 2000, 114: 1 9.

[6] Liu Shulin, Qiu Wanhua, Studies on the Basic Theories for MADM. System Engineering-Theory \& Practice. 1998, 1: 38 43.

[7] Liou T S, Wang M J J. Ranking fuzzy numbers with integral value. Fuzzy sets and systems, 1992, 50: 247 255.

[8] R. J. Li and E. S. Lee, Comparison of Fuzzy numbers based on the probability measure of fuzzy events. Comp. Math. Applic, 1987, 15: 887 896.

[9] R. Csutoraa, J.J.Buckleyb. Fuzzy hierarchical analysis: The Lambda-Max method. Fuzzy Sets and Systems, 2001, 120: 181-195.

[10] J.J. Buckley, T. Feuring, Y. Hayashi, Fuzzy hierarchical analysis revisited. European Journal of Operational Research, 2001, 129: 48-64.

[11] N. Bryson, Group decision-making and the analytic hierarchy process: Exploring the consensus-relevant information content. Computer \& Operations research. 1996, 23: $27 \sim 35$.

[12] J.S. Dyer, A clarification of "Remarks on the analytic hierarchy process". Management Science, 1990, 36: 274 275.

[13] T.L. Saaty Highlights and critical points in the theory and application of analytic hierarchy process. European Journal of Operational Research, 1997, 74: 426 447

[14] T.L. Saaty, Rank generation, preservation, and reversal in the analytic hierarchy process. Decision Sic., 1987, 18: 157 177.

[15] Fong-Gong Wu, Ying-Jye Lee, Ming-Chyuan Lin, Using the fuzzy analytic hierarchy process on optimum spatial allocation. International Journal of Industrial Ergonomics, 2004, 33: 553-569.

[16] Zhang jijun. Fuzzy analytic hierarchy process, Chinese Journal of Fuzzy systems and mathematics, 1999, 14: 81 89.

[17] R. J. Li. FMCDM: Theories and Applications. Beijing, Science Press, 2002.

[18] Kong feng, Liu hongyan. Fuzzy Entropy-Weight Algorithm for Multiple Attribute Decision Making Based on Uncertainness Preference. Proceedings of the Seventh International Conference on Industrial Management, Beijing, 2004: 616 621.

[19] Carlsson C, Fuller R.. Fuzzy multiple criteria decision making: recent developments. Fuzzy sets and Systems, 1996, 78: 139 153.

[20] E. S. Lee and R. J. Li. Comparison of Fuzzy numbers based on the probability measure of fuzzy events. Comp. Math. Applic. 1987, 15: 887 896.

[2I] R. M. Tong, P.P Bonissone. A linguistic approach to decision making with fuzzy sets. IEEE Trans. System Man Cybernet, 1980, 10: 716 723.

[22] J. Buckley. Ranking alternatives using fuzzy numbers. Fuzzy sets and systems, 1985, 15: $21-34$. 